\title{
Blueshift of photoluminescence peak in ten periods InAs quantum dots superlattice
}

\author{
Ray-Ming Lin ${ }^{\mathrm{a}, *}$, Si-Chen Lee ${ }^{\mathrm{b}}$, Hao-Hsiung Lin ${ }^{\mathrm{b}}$, Yuan-Tung Dai ${ }^{\mathrm{c}}$, \\ Yang-Fang Chen $^{\mathrm{c}}$ \\ ${ }^{a}$ Department of Electronic Engineering, Chang Gung University, Tao-Yuan, Taiwan \\ ${ }^{\mathrm{b}}$ Department of Electrical Engineering, National Taiwan University, Taipei, Taiwan \\ ${ }^{\mathrm{c}}$ Department of Physics, National Taiwan University, Taipei, Taiwan
}

\begin{abstract}
We have observed a temperature insensitive and an unusual blueshift of photoluminescence peak energy in ten periods self-organized InAs quantum dots superlattice. The net temperature dependence of PL wavelength for samples with 3, 4 and 5 monolayer ten periods InAs quantum dots superlattice, respectively, are $0.15,0.13$ and $-0.17 \mathrm{~nm} /{ }^{\circ} \mathrm{C}$, respectively. The temperature dependence of the InAs quantum dots shows a significantly different trend when the InAs layer thickness is smaller (redshift) or larger (blueshift) than around 4 ML. (C) 2001 Elsevier Science B.V. All rights reserved.
\end{abstract}

PACS: $68.55 . \mathrm{Bd} ; 68.55 . \mathrm{JK} ; 68.10 . \mathrm{Cr}$

Keywords: A1. Quantum dots; A3. Molecular beam epitaxy; B2. Semiconducting indium compounds; B3. Nonlinear optical devices

\section{Introduction}

It was found from both theory and experiment that the magnitude of temperature effects on the bandgap energies of a biaxially strained heterostructure is about the same as that of bulk material [1]. If no other mechanisms are involved, the photoluminescence (PL) peak of the bulk and the biaxially strained structure should have shown a substantial redshift on increasing the temperature. In long wavelength GaInAs/InP lasers, it is known that the typical value of temperature sensitivity for the lasing wavelength is about $0.5 \mathrm{~nm} /{ }^{\circ} \mathrm{C}$ [2] and the wavelength shift in InAs bulk material is about

*Corresponding author.Tel.: + 886-3-328-3016; fax; + 886-3328-2107.

E-mail address: rmlin@mail.cgu.edu.tw (R.-M. Lin).
$0.93 \mathrm{~nm} /{ }^{\circ} \mathrm{C}$ in the temperature range from 0 to $80 \mathrm{~K}$ [3].

Low dimensional semiconductor structures constitute very attractive objects both for their fundamental properties and their potential application in micro- and optoelectronics [4]. Spontaneous formation of three-dimensional (3D) islands during Stranski-Krastanov-like growth of highly strained InAs layers on GaAs substrates by molecular beam epitaxy (MBE) has been proposed as a promising way for fabricating high-quality InAs quantum dots [5-7]. When the thickness of an InAs layer grown on GaAs is beyond a critical thickness $\left(L_{\mathrm{c}}\right)$, the structure is usually converted from an InAs wetting layer to a dislocation free coherent island. Leonard et al. reported the formation of dislocated islands, when the amount of InAs grown on GaAs exceeded 2 monolayers 
(ML) [8,9]. $\mathrm{Xu}$ et al. observed an unusual temperature dependence of exciton energy in InAs quantum dots grown on GaAs [10]. The rate of energy redshift with temperature becomes much greater than the InAs bandgap variation with temperature. They believe that this unusual bandgap variation with temperature in self-organized InAs quantum dots results from the enhanced carrier relaxation process related to the size distribution of quantum dots.

Cheng et al. [11] reported on the PL properties of $\mathrm{Ga}_{x} \mathrm{In}_{1-x}$ As strained multiple-quantum-wire heterostructures formed in situ by the straininduced lateral-layer ordering process and proposed the thermal expansion effect as the most likely explanation for the temperature invariant and even a blueshift of PL spectra.

In this paper, we have successfully grown the high quality ten periods 3,4 and $5 \mathrm{ML}$ InAs quantum dots on GaAs substrates and systematically studied the temperature dependence of the PL spectra. Meanwhile, we have observed a temperature insensitive, unusual blueshift of PL peak energy in the ten periods InAs quantum dots superlattice. In addition, we propose one possible explanation to interpret the unusual blueshift of PL peak energy in the ten periods $5 \mathrm{ML}$ InAs quantum dots superlattice.

\section{Experiments}

The samples were grown on semi-insulating undoped (100) on-axis GaAs substrates by VG V80 H MK II solid source MBE equipped with a $15 \mathrm{kV}$ reflection high energy electron diffraction (RHEED) system. First, a $300 \mathrm{~nm}$ GaAs buffer layer was deposited at $580^{\circ} \mathrm{C}$, then a single layer of quantum dots was grown by depositing InAs of nominal thickneses of 3,4 and $5 \mathrm{ML}$ InAs at $500^{\circ} \mathrm{C}$, respectively, by a migration enhanced technique and the growth rate was kept at $0.53 \mu \mathrm{m} / \mathrm{h}$. The RHEED pattern shows a transition from streaky to well developed diffraction spots as a result of a $3 \mathrm{D}$ growth mode. During the growth of the subsequent layers (10 ML GaAs), the RHEED pattern is recovered with streaky pattern and shows a transition from 3D growth mode to a two dimensional (2D) growth mode. The ten periods superlattice was obtained by repeating the single layer of quantum dots growth procedure ten times and the InAs quantum dots are separated by a $10 \mathrm{ML}$ GaAs.

For a PL measurement, the signals were generated by an Ar ion laser. The sample was placed in an Oxford liquid helium chamber. The luminescence signal was dispersed by an SPEX $0.5 \mathrm{~m}$ monochromator and detected by a liquid nitrogen cooled North-Coast germanium detector.

\section{Results and discussion}

Fig. 1 shows the temperature dependence (5-80 K) of the PL spectra with $3 \mathrm{ML}$ InAs quantum dots superlattice. The transition energies determined by the peak position of PL are $1.162 \mathrm{eV}(5 \mathrm{~K}), 1.159 \mathrm{eV}(10 \mathrm{~K}), 1.155 \mathrm{eV}(20 \mathrm{~K})$, $1.152 \mathrm{eV}(50 \mathrm{~K})$ and $1.148 \mathrm{eV}(80 \mathrm{~K})$, and the corresponding values of full width at half-maximum (FWHM) are 33, 44, 41, 37 and $45 \mathrm{meV}$, respectively. Fig. 2 shows the temperature dependence $(5-80 \mathrm{~K})$ of the PL spectra with $4 \mathrm{ML}$ InAs quantum dots superlattice. The transition energies also determined by the peak positions of PL are $1.140 \mathrm{eV}(5 \mathrm{~K}), 1.138 \mathrm{eV}(10 \mathrm{~K}), 1.136 \mathrm{eV}(20 \mathrm{~K})$, $1.132 \mathrm{eV}(50 \mathrm{~K})$ and $1.128 \mathrm{eV}(80 \mathrm{~K})$, and the corresponding values of FWHM are 45, 45, 45, 44 and $40 \mathrm{meV}$, respectively. Fig. 3 shows the temperature dependence $(5-80 \mathrm{~K})$ of the $\mathrm{PL}$

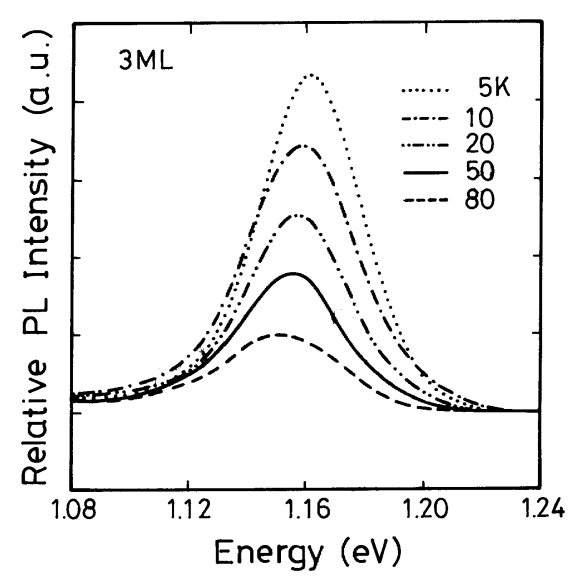

Fig. 1. Temperature dependence of PL spectra with ten periods $3 \mathrm{ML}$ InAs quantum dots superlattice grown on GaAs. 


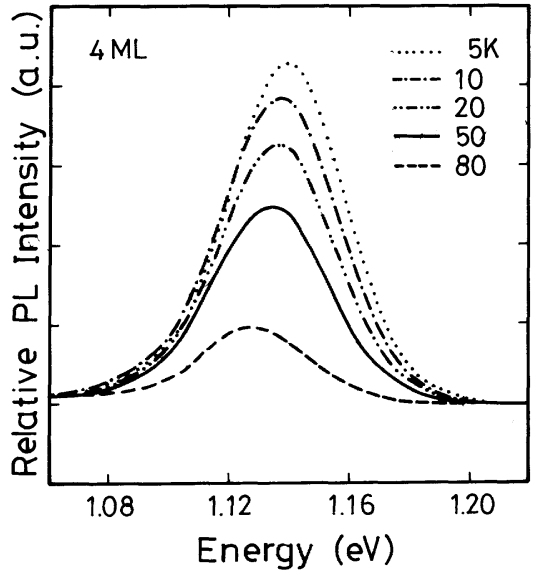

Fig. 2. Temperature dependence of PL spectra with ten periods 4 ML InAs quantum dots superlattice grown on GaAs.

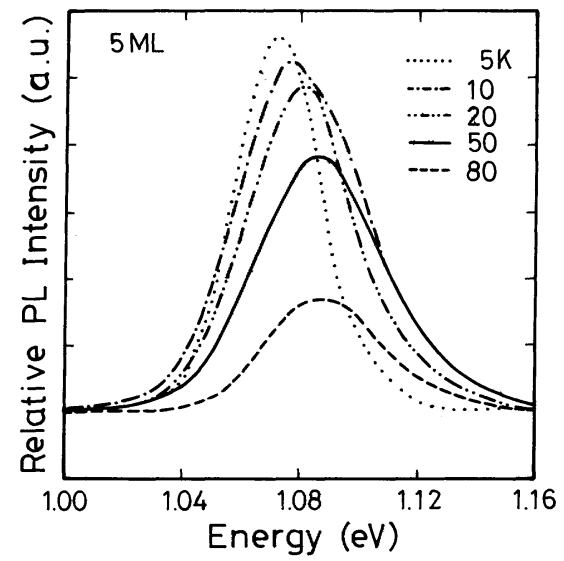

Fig. 3. Temperature dependence of PL spectra with ten periods 5 ML InAs quantum dots superlattice grown on GaAs.

spectra with $5 \mathrm{ML}$ InAs quantum dots superlattice. The transition energies determined by the peak positions of PL are $1.074 \mathrm{eV}(5 \mathrm{~K}), 1.075 \mathrm{eV}$ $(10 \mathrm{~K}), \quad 1.081 \mathrm{eV}(20 \mathrm{~K}), \quad 1.088 \mathrm{eV} \quad(50 \mathrm{~K})$ and $1.090 \mathrm{eV}(80 \mathrm{~K})$, and the corresponding values of FWHM are 35, 43, 48, 49 and $44 \mathrm{meV}$, respectively. The FWHM values of the three samples are all less than $50 \mathrm{meV}$ at various temperatures, indicating a more uniformly distributed quantum size $[12,13]$. We believe that this better uniformity in the ten periods InAs quantum dots superlattice is due to a migration enhanced growth technology during MBE.

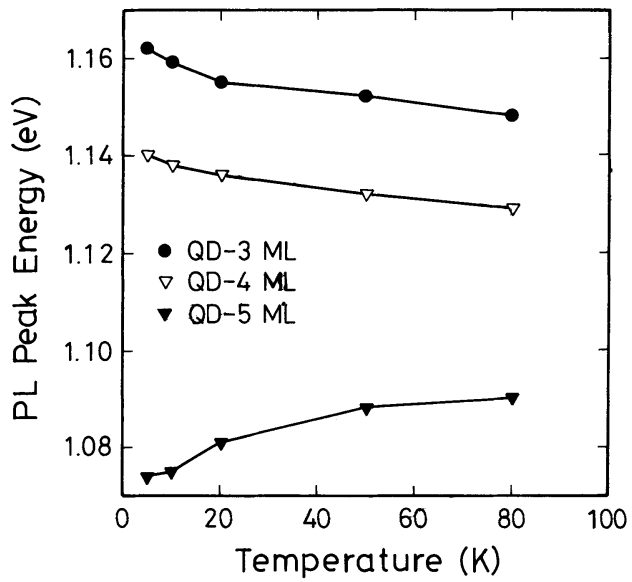

Fig. 4. Temperature dependence of the PL peak energy for the ten periods 3,4 and $5 \mathrm{ML}$ InAs quantum dots superlattice grown on GaAs.

Fig. 4 presents the temperature dependence of the PL peak energy of different InAs samples. It is clear that the energy variation with temperature could be divided into two categories: when the InAs layer is smaller than or equal to $4 \mathrm{ML}$, the emitted photon energy of the InAs layer redshifts with increasing temperature. However, when the layer thickness becomes larger than $5 \mathrm{ML}$, the emitted photon energy of the InAs layer blueshifts with increasing temperature. The rate of energy shift with temperature for the ten periods InAs quantum dots superlattice structure with 3,4 and $5 \mathrm{ML}$, are about $0.15,0.13$ and $-0.17 \mathrm{~nm} /{ }^{\circ} \mathrm{C}$, respectively. As mentioned above, the wavelength shift in InAs bulk materials is about $0.93 \mathrm{~nm} /{ }^{\circ} \mathrm{C}$. So using quantum dots as an active region, a laser with a temperature-insensitive stimulated emission wavelength could be fabricated.

The redshift of a semiconductor with increasing temperature is known to be mainly due to thermal expansion of the lattice constant and electronphonon scattering [14]. Also, band filling effect in $\mathrm{k}$-space can contribute a blueshift to the bandgap energy, but due to a slight nonuniformity in dot or wire size, the sharp density of states function will be broadened and show a more quantum welllike temperature dependence $[1,11]$. From the experimental results, there are reasonable explanations to interpret the unusual blueshift of PL peak 


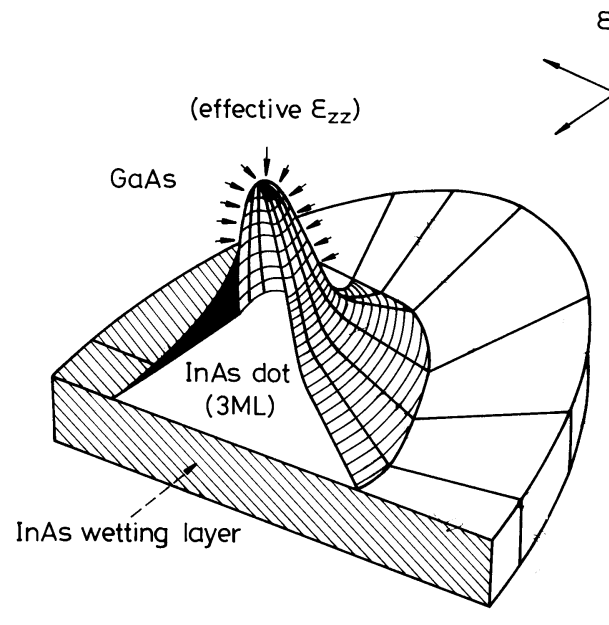

(a)

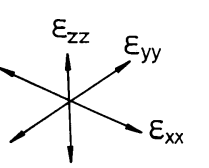

(effective $\varepsilon_{z z}$ )

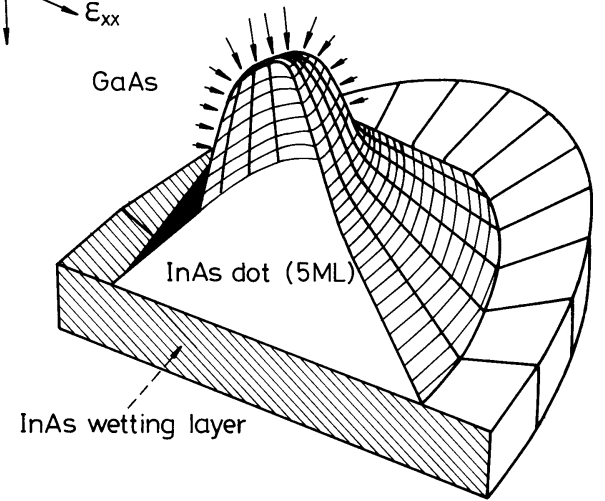

(b)

Fig. 5. An illustration of the triaxial strain in a self-organized InAs dots region with InAs (a) $3 \mathrm{ML}$, and $5 \mathrm{ML}$ thick.

energy in the ten periods $5 \mathrm{ML}$ InAs quantum dots superlattice.

The evolution of a random initial surface according to the Huygens principle, an initially rough interface grows with a constant velocity along the local surface normal [15] and Chang has studied the shapes of $1,2,3,4,5,6,10$, and 15 ML InAs, respectively, with InAs/GaAs multilayer structure by high resolution transmission electron microscopy (HRTEM) and reported that the larger the InAs dot, the flatter is the top of the island [16]. Fig. 5 illustrates the shape and the triaxial strain of two self-organized InAs dots with 3 ML (Fig. 5(a)) and 5 ML (Fig. 5(b)) thickness. For InAs grown on GaAs, it is clear that variation of the emission energy with temperature will be affected by two different strained regions, i.e., dots which are in contact with the InAs wetting layer (region I) and those in contact with the surrounding GaAs layer area (region II), as shown in Fig. 5. An InAs wetting layer with compressive strains $\left(e_{x x}\right.$ and $\left.e_{y y}\right)$ at the hetero-interface will have the crystal lattice in the growth direction $(z)$ free to expand with temperature, which determines the conduction band minimum and thus the PL peak energy. In the conelike InAs dot region, there exist not only the biaxial compressive strain $\left(e_{x x}\right.$ and $\left.e_{y y}\right)$ at the interfaces between the InAs wetting layer and the cone (region I), but also an additional strain $\left(e_{z z}\right)$ between the InAs dot and the surrounding GaAs region (region II) in the growth direction. For $5 \mathrm{ML}$ dots, the top of the island is flatter as shown in Fig. 5(b). So there is very little interface stress in the direction $(z)$ normal to the dot surface, especially in the central region of the island. The lattice is free to expand in the $z$ direction, resulting in a larger $e_{z z}$ and thus lower conduction band minimum. When the temperature increases from $\sim 5$ to $80 \mathrm{~K}$, the crystal lattice of all regions tends to expand. Due to the difference of thermal expansion coefficients of the two materials, i.e., InAs has a smaller expansion coefficient than that of GaAs, the compressive strain $\left(e_{x x}\right.$ and $\left.e_{y y}\right)$ of InAs island will be reduced when the temperature increases, and so will $e_{z z}$. For the $5 \mathrm{ML}$ sample, the change of $e_{z z}$ is the largest.

As shown in Fig. 5(b), the larger the InAs dot size, the more moderate is its dot shape, and thus it induces more strain relaxation in the dot region. In addition, the more moderate dot shape (flat top surface) will affect the weighting of multiaxial strain in the InAs dot region, i.e., the strain $e_{z z}$ is larger. So in self-organized InAs quantum dots, enlarging the dot size will not only induce more strain relaxation but also result in a more moderate dot shape, where the former tends to reduce but the latter tends to increase the strain $e_{z z}$ in the direction normal to the growth plane. When 
the sample is heated from $\sim 5$ to $80 \mathrm{~K}$, the decrease of in plane thermal expansion strain $\left(e_{x x}\right.$ and $\left.e_{y y}\right)$ in the InAs quantum dot region also reduces $e_{z z}$ which in turn increases the conduction band minimum and thus the PL peak energy. The increment of bandgap of the $5 \mathrm{ML}$ sample definitely compensates the narrowing of bandgap due to lattice expansion as temperature increases which leads to the observed blueshifts of PL peak energy. For samples with 3 and $4 \mathrm{ML}$, PL peak energies display an insensitive redshift with increasing temperature indicating the reduction of $e_{z z}$ due to less interface mismatch overcompensated by the lattice expansion.

\section{Conclusion}

We have observed a temperature insensitive, unusual blueshift of PL peak energy in ten periods InAs quantum dots superlattice with respective 3, 4 and $5 \mathrm{ML}$ InAs as the well region grown on GaAs. The net temperature dependence of PL wavelength for ten periods InAs quantum dots superlattice with 3,4 and $5 \mathrm{ML}$, are $0.15,0.13$ and $-0.17 \mathrm{~nm} /{ }^{\circ} \mathrm{C}$, respectively. So using the quantum dots as an active region, a laser with a temperature-insensitive stimulated emission wavelength could be fabricated. For the ten periods $5 \mathrm{ML}$ InAs quantum dots superlattice, the PL peak wavelength was found to blueshift with increasing temperature. It is believed that the flatter top surface of $5 \mathrm{ML}$ dots is responsible for the larger variation of $e_{z z}$ which has compensated the temperature induced lattice expansion, and results in the blueshift of PL peak energy.

\section{Acknowledgements}

The authors are grateful to Professor Tzer-En Nee for helpful discussion. This work was supported by the National Science Council of the Republic of China under Contract No. NSC 89-2215-E-182-005.

\section{References}

[1] Y. Miyake, H. Hirayama, K. Kudo, S. Tamura, A. Arai, M. Asada, U. Miyamoto, Y. Suematsu, IEEE J. Quantum Electron. 29 (1993) 2123.

[2] G.P. Agrawal, N.K. Dutta, Long Wavelength Semiconductor Lasers, 2nd Edition, Van Nostrand Reinhold, New York, 1993 (Chapter 7).

[3] J. Singh, Physics of Semiconductors and Their Heterostructures, McGraw-Hill, New York, 1993 (Chapter 5).

[4] Y. Nabetani, T. Ishikawa, S. Noda, A. Sasaki, J. Appl. Phys. 76 (1994) 347.

[5] J.M. Moison, F. Houzay, F. Barthe, L. Leeprince, E. Andre, O. Vatel, Appl. Phys. Lett. 64 (1994) 196.

[6] J.Y. Marzin, J.M. Gerard, A. Izrael, D. Barrier, G. Bastard, Phys. Rev. Lett. 73 (1994) 716.

[7] J.I. Chyi, T.E. Nee, C.T. Lee, J.L. Shieh, J.L. Shieh, J.W. Pan, J. Crystal Growth 175/176 (1997) 777.

[8] D. Leonard, K. Pond, P.M. Petroff, Phys. Rev. B 50 (1994) 11687.

[9] Mark S. Miller, Jan-Olle Malm, Mats-Erik Pistol, Soren Jeppesen, Bernhard Kowalski, Kristina Georgsson, Lars Samuelson, J. Appl. Phys. 80 (1996) 3360.

[10] Z.Y. Xu, Z.D. Lu, X.P. Yang, Z.L. Yuan, B.Z. Zheng, J.Z. Xu, W.K. Ge, Y. Wang, L.L. Chang, Phys. Rev. B 54 (1996) 11528.

[11] D.E. Wohlert, S.T. Chou, A.C. Chen, K.Y. Cheng, K.C. Hsieh, Appl. Phys. Lett. 68 (1996) 2386.

[12] D.I. Lubyshev, P.P. Gonalez-Borrero, E. Marega Jr., E. Petitprez, N. La Scala Jr., P. Basmaji, Appl. Phys. Lett. 68 (1996) 205.

[13] D. Leonard, S. Fafard, K. Pond, Y.H. Zhang, J.L. Mertz, P.M. Petroff, J. Vac. Sci. Technol. B 12 (1994) 2516.

[14] D. Gammon, S. Rudin, T.L. Reincke, D.S. Katzer, C.S. Kyono, Phys. Rev. B 51 (1995) 16785.

[15] C. Tang, S. Alexander, R. Bruinsma, Phys. Rev. A 31 (1990) 772.

[16] T.C. Chang, Transmission electron microscopy studies of InGaAs/GaAs heteroepitaxy grown by molecular beam epitaxy, Master Thesis, National Taiwan University, Taipei, Taiwan, R O C, 1992. 